\title{
Review
}

\section{Status of antituberculosis drug resistance in Saudi Arabia 1979-98}

\author{
K.K. Abu-Amero'
}

SUMMARY Al published material on the prevalence of drug-resistant tuberculosis within Saudi Arabia over the period $1979-98$ was reviewed. The prevalence of single-drug-resistant tuberculosis ranged from $3.4 \%$ to $41 \%$ for isoniazid, $0 \%$ to $23.4 \%$ for rifampicin, $0.7 \%$ to $22.7 \%$ for streptomycin and $0 \%$ to $6.9 \%$ for ethambutol. The prevalence of multidrug-resistant tuberculosis (defined by WHO as resistance to two or more firstline antituberculosis drugs) ranged from $1.5 \%$ to $44 \%$ in different regions. No strong conclusions could be drawn owing to variations in the populations studied, geographical origins, site of Mycobacterium tuberculosis isolation (pulmonary or extrapulmonary) and drug sensitivity testing. However, the need to develop a standardized national policy for surveillance of drug-resistant tuberculosis in Saudi Arabia is clear.

\section{Introduction}

After years of decline, tuberculosis (TB) has re-emerged as a serious public health problem worldwide. In 1993 the World Health Organization (WHO) declared it a global emergency: according to a recent WHO report, there were 7.96 million new cases in 1997 with 2 million deaths [1]. Factors contributing to the resurgence of TB include the human immunodeficiency virus epidemic, immigration of persons from countries with a high incidence of TB, and political turmoil in some developing countries, which has hindered efforts to control the disease.

The re-emergence of TB is a public health concern, especially with increased drug resistance among Mycobacterium tuberculosis strains, which have acquired varying degrees of resistance to first-line antituberculosis drugs. It has been suggest- ed that the underlying causes of singledrug-resistant TB (SDR-TB) and/or multidrug-resistant TB (MDR-TB) may be incorrect treatment, poor compliance with treatment regimens, shortage of antituberculosis drugs due to financial constraints in some developing countries, and deficient or deteriorating $\mathrm{TB}$ control programmes, resulting in inadequate administration of effective chemotherapy $[2,3]$. Previous reports indicate that SDR-TB and MDR-TB are on the increase in both industrialized and developing countries $[4,5]$.

A recent WHO report presented global data on the prevalence of drug-resistant TB for the period 1996-99 [6] (see Table 1). The prevalence of SDR-TB ranged from $2.9 \%$ in New Caledonia to $40.8 \%$ in Estonia. MDR-TB prevalence ranged from $0 \%$ in Finland and New Caledonia to $18.1 \%$ in Estonia. Eight countries showed a prevalence of MDR TB greater than $5 \%$. Of the

'Department of Biological and Medical Research, King Faisal Specialist Hospital and Research Centre, Riyadh, Saudi Arabia.

Received: 25/06/01; accepted: 08/01/02 
Table 1 Summary of the WHO Report[G] on the prevalence of drug-resistant tuberculosis (1996-99)

\begin{tabular}{|c|c|c|c|c|c|c|c|}
\hline Country & Year & $\begin{array}{c}\text { SDR-TB } \\
\text { (\% of } \\
\text { cases) }\end{array}$ & $\begin{array}{c}\text { MDR-TB } \\
\text { (\% of } \\
\text { cases) }\end{array}$ & Country & Year & $\begin{array}{c}\text { SDR-TB } \\
\text { (\% of } \\
\text { cases) }\end{array}$ & $\begin{array}{c}\text { MDR-TB } \\
\text { (\% of } \\
\text { cases) }\end{array}$ \\
\hline Australia & 1996 & 10.5 & 2.0 & Mozambique & 1999 & 23.1 & 3.5 \\
\hline Belgium & 1997 & NR & 2.0 & Nepal & 1999 & 6.4 & 1.4 \\
\hline Botswana & 1999 & 7.7 & 1.2 & Netherlands & 1996 & 11.0 & 0.6 \\
\hline Canada & 1997 & 10.5 & 1.1 & New Caledonia & 1996 & 2.9 & 0 \\
\hline Central African & & & & New Zealand & 1997 & 12.0 & 1.0 \\
\hline Republic & 1998 & 18.4 & 2.8 & Norway & 1996 & 11.1 & 2.8 \\
\hline Chile & 1997 & 10.5 & 1.1 & Pent & 1999 & 18.8 & 4.3 \\
\hline China & $996-99$ & 23.4 & 7.6 & Poland & 1997 & 5.1 & 1.3 \\
\hline Cuba & 1998 & 8.3 & 0.9 & Puerto Rico & 1997 & 14.5 & 3.5 \\
\hline Czech Republic & 1999 & 4.2 & 2.0 & Republic & & & \\
\hline Denmark & 1998 & 13.1 & 0.7 & of Korea & 1999 & 11.8 & 2.7 \\
\hline $\begin{array}{l}\text { England } \\
\text { and Wales }\end{array}$ & 1997 & 8.1 & 1.5 & $\begin{array}{l}\text { Russian } \\
\text { Federation }\end{array}$ & $1998-99$ & 39.4 & 13.0 \\
\hline Estonia & 1998 & 40.8 & 18.1 & Scotiand & 1997 & 4.2 & 0.7 \\
\hline Fintand & 1999 & 4.9 & 0 & Sierra Leone & 1997 & 27.7 & 2.6 \\
\hline France & 1997 & 11.1 & 0.4 & Singapore & 1996 & 5.9 & 0.8 \\
\hline Germany & 1998 & 10.2 & 1.7 & Slovakia & 1998 & 5.5 & 2.0 \\
\hline Guinea & 1998 & 15.9 & 1.5 & Slovenia & 1997 & 3.1 & 0.9 \\
\hline India (Tamil & & & & South Africa & 1997 & 10.2 & 2.5 \\
\hline Nadu state) & 1997 & 24.1 & 7.1 & Spain & 1998 & 5.4 & 1.4 \\
\hline Islamic Republic & & & & Sweden & 1997 & 8.4 & 1.1 \\
\hline of Iran & 1998 & 17.6 & 6.7 & Switzerland & 1997 & 5.8 & 1.4 \\
\hline Israel & 1998 & 19.2 & 8.1 & Uganda & 1997 & 22.1 & 0.8 \\
\hline Italy & 1999 & 20.5 & 6.7 & United States & & & \\
\hline Latvia & 1998 & 30.1 & 12.0 & of America & 1997 & 12.4 & 1.4 \\
\hline Malaysia & 1997 & 5.1 & 0.1 & Uruguay & 1997 & 4.6 & 0.2 \\
\hline Mexico & 1997 & 20.6 & 7.3 & Venezuela & 1998 & 4.7 & 0.4 \\
\hline
\end{tabular}

SDR-TB = single-drug-resistant tuberculosis.

MDR-TB = multidrug-resistant tuberculosis

$N R=$ not reported.

countries that provided two or more data points, Germany $(7.7 \%$ versus $10.2 \%$ ) and New Zealand $(4.8 \%$ versus $12.0 \%)$ reported statistically significant increases $(P<$
$0.001)$ in the prevalence of any drug-resistant TB in the most recent year for which surveillance data were available, compared with the previous year. The Netherlands,

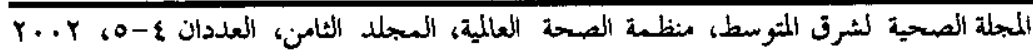


on the other hand, reported a significant downward trend $(14.1 \%$ versus $11.0 \%, P$ $=0.02$ ).

The prevalences of SDR-TB and MDRTB in Saudi Arabia are largely unknown, as only limited-scale studies with variable drug resistance methods were used. In addition, the WHO report on antituberculosis drug resistance did not include data from Saudi Arabia [6]. The objective of this study was to review all published material on the prevalence of SDR-TB and MDRTB for different regions of Saudi Arabia, to determine the status of antituberculosis drug resistance in the country. Possible reasons for the current status of drug resistance in Saudi Arabia are also discussed.

\section{Methods}

A MEDLINE search of the world literature from 1966 to September 2001 was performed using 'tuberculosis' and 'Saudi Arabia' as key words. Any surveys of antimicrobial susceptibility testing of $M$. tuberculosis isolates performed on isolates from Saudi Arabia were identified. Advice was sought from the Ministry of Health in Riyadh, who is responsible for collecting data on the incidence of TB and drug-resistant status.

For single drugs, the percentage of isolates that were resistant to the drug were recorded. Multidrug resistances reported here were for isolates that were resistant to two or more first-line antituberculosis drugs, according to WHO guidelines [7]. Where particular data were not reported, this was recorded as such. All isolates reported here represent one patient.

\section{Results}

Analysis of reports from different areas of Saudi Arabia indicate that the percentage of isolates resistant to isoniazid, as a single drug, were $3.4 \%-19.4 \%$ in Riyadh (located in the central province of Saudi Arabia), $10.3 \%-28.7 \%$ in Jeddah (located in the western province of Saudi Arabia), $6 \%$ in Dammam (located in the eastern province of Saudi Arabia), $41 \%$ in Gizan (located in the southern province of Saudi Arabia) and $6.5 \%$ in Taif (located in the southwest of Saudi Arabia). Resistances to rifampicin, as a single drug, were $0 \%-9.7 \%, 5.1 \%$ $23.4 \%, 0.2 \%, 20 \%$ and $15.3 \%$ for Riyadh, Jeddah, Dammam, Gizan and Taif respectively. Resistances to streptomycin, as a single drug, were $0.75 \%-12.5 \%, 7.7 \%$ $22.7 \%, 0.7 \%, 26 \%$ and $15.9 \%$ for Riyadh, Jeddah, Dammam, Gizan and Taif respectively. Resistances to ethambutol, as a single drug, were $2.0 \%-2.4 \%, 1.3 \%-6.9 \%$, $3.9 \%$ and $4 \%$ for Riyadh, Jeddah, Gizan and Taif respectively. However, $0 \%$ resistance to ethambutol was reported from the Dammam area [8] (Table 2). Within SDR$\mathrm{TB}$, resistance to isoniazid $(3.4 \%-41.0 \%)$ was the most common, followed by resistance to rifampicin $(0 \%-23.4 \%)$, then streptomycin $(0.7 \%-22.7 \%)$ and finally ethambutol $(0 \%-6.9 \%)$.

For MDR-TB, reported resistances were $1.5 \%-11.8 \%, 11.5 \%-25.7 \%, 10.5 \%$, $44 \%$ and $15.3 \%$ for Riyadh, Jeddah, Dammam, Gizan and Taif respectively (Table 2 ). The majority of reports found isoniazid and rifampicin to be the most resistant combination, followed by the combination isoniazid, rifampicin and streptomycin [9].

\section{Discussion}

Saudi Arabia, with an area of 865000 square miles, occupies the bulk of the Arabian peninsula. It is roughly one-third of the size of the United States of America, and the size of western Europe. It is also one of the richest countries in the world,

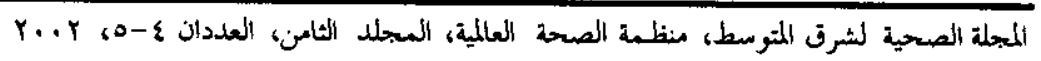


Table 2 Summary of studies on antituberculosis drug resistance among Mycobacterium tuberculosis isolates from different reglons of Saudi Arabia 1979-98

\begin{tabular}{|c|c|c|c|c|c|c|c|c|}
\hline \multirow[b]{2}{*}{ Location } & \multirow{2}{*}{\multicolumn{2}{|c|}{ Year(s) }} & \multirow[b]{2}{*}{$n$} & \multicolumn{4}{|c|}{ SDR-TB (\% of cases) } & \multirow{2}{*}{$\begin{array}{c}\text { MDR-TB } \\
\text { (\% of cases) }\end{array}$} \\
\hline & & & & Isonlazld & Rifampicin & Streptomycin & Ethambutol & \\
\hline Riyadh & $1979-82$ & {$[10]$} & 1968 & 4.2 & NR & 12.5 & NR & 3.7 \\
\hline Riyadh & $1981-82$ & [11] & 47 & 7 & 9 & NR & 2 & 10.6 \\
\hline Gizan & 1985 & {$[12]$} & 108 & 41 & 20 & 26 & 3.9 & 44 \\
\hline Taif & $1986-88$ & {$[13]$} & 678 & 6.5 & 15.3 & 15.9 & 4 & 15.3 \\
\hline Riyadh & $1986-88$ & {$[14]$} & 432 & 19.4 & 9.7 & 5.1 & NA & 11.8 \\
\hline Riyadh & $1987-97$ & {$[15]$} & 670 & 3.4 & 0 & 0.75 & 0 & 1.5 \\
\hline Riyadh & $1989-94$ & {$[16]$} & 289 & 7.2 & 3.1 & 3.5 & 2.4 & 3.8 \\
\hline Jeddah & 1991 & {$[17]$} & 483 & 17.8 & 23.4 & 16.4 & 3.9 & 17.6 \\
\hline Jeddah & 1993-95 & {$[18]$} & 78 & 10.3 & 5.1 & 7.7 & 1.3 & 11.5 \\
\hline Dammam & $1993-96$ & {$[8]$} & 411 & 6 & 0.2 & 0.7 & 0 & 10.5 \\
\hline Jeddah & $1996-98$ & {$[19]$} & 101 & 28.7 & 20.7 & 22.7 & 6.9 & 25.7 \\
\hline
\end{tabular}

SDR-TB = single-drug-resistant tuberculosis.

MOR-TB = multidrug-resistant tuberculosis (defined by WHO as tuberculosis that is resistant to two or more first-line antituberculosis drugs).

NR $=$ not reported.

$n=$ number of isolates or patients studied.

with a comprehensive health care system, which currently has 306 state-run hospitals and 1700 state-run primary health care centres scattered throughout the country.

This review has achieved comprehensive coverage of all possible sources of information about SDR-TB and MDR-TB in Saudi Arabia, from the Internet and other literature searches and from the Ministry of Health (who had no data to add to that published in the literature).

Reports on antituberculosis drug resistance from different regions of Saudi Arabia give a contradictory picture of the status of drug-resistant TB in the country. Reports from Jeddah showed a steady increase in the prevalence of MDR-TB, which increased almost threefold during the period 1991-98 [19]. Suggestions for the cause of the high incidence of drug resistance around Jeddah include exposure of the local population to expatriate workers, illegal immigrants and pilgrims travelling to Mecca $[18,20]$. Each year around two million pilgrims from all parts of the world pass through Jeddah on their way to Mecca to perform Hajj; most are from poor countries with a high prevalence of infectious diseases such as TB. A recent report has shown that TB was the most common cause of pneumonia requiring hospitalization among the pilgrims in Mecca [21]. Resistance to isoniazid, as a single drug, was the most common, followed by resistance to rifampicin. Similar patterns of high resistance to isoniazid were seen throughout Saudi Arabia (Table 2) [9,22]; this could be a result of non-compliance with treatment regimens or misuse of the drug for chemoprophylaxis [23].

In Riyadh, the prevalence of MDR-TB ranged from $1.5 \%$ to $11.8 \%$ over the period

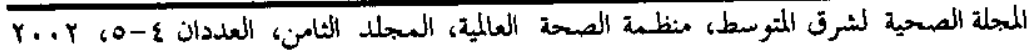


1979-97 (Table 2). In one of these studies, conducted at the Riyadh Armed Forces Hospital over the period 1987-97 [15], the authors reported a very low MDR-TB rate of $1.5 \%$. They attributed this to the fact that their study sample consisted mainly of natives, and suggested that the source of highly resistant tubercle bacilli, seen in previous studies from Saudi Arabia, could be expatriates, especially those from the Indian subcontinent. This conclusion was substantiated by two reports from the Riyadh area, which covered natives and the expatriate population and reported relatively high MDR-TB rates of $10.6 \%$ and $11.8 \%$ $[11,14]$.

There was one report from Taif [13] that showed a high rate of MDR-TB (15.3\%) compared with rates in other parts of the country. This high rate in Taif was attributed to possible non-compliance with antituberculosis treatment. This was later confirmed by Al-Hajjaj and Al-Khatim (2000) [23], who investigated the problem of non-compliance thoroughly. They concluded that non-compliance with antituberculosis treatment, despite a retrieval system in operation, was the major cause of the high MDR-TB rate, and called for urgent implementation of a DOTS (directly observed treatment, short course) system in Saudi Arabia.

In Dammam, there was one report concerning MDR-TB [8], which covered the period 1993-96. The prevalence of MDRTB reported from this area was $10.5 \%$, which is comparable to rates of MDR-TB reported from other parts of Saudi Arabia.

In Gizan, the MDR-TB rate was extremely high (44\%) [12] compared with MDR-TB rates reported from other regions of the country [24]. The investigators attributed this to the close proximity of Gizan to the Republic of Yemen, which has been reported to have one of the highest rates of active TB of all Arab countries, and to the movement of labourers across the border, who bring MDR-TB with them [25].

For SDR-TB, the prevalence in Saudi Arabia for the period 1979-98 ranged from $0 \%$ to $41 \%$ and the highest resistance reported for a single drug was for isoniazid. In comparison, the prevalence of SDR-TB globally, reported recently by WHO [6], ranged from $4.6 \%$ to $18.8 \%$ (South America), $4.2 \%$ to $40.8 \%$ (Europe), $8.3 \%$ to $20.6 \%$ (North America), $15.9 \%$ to $27.7 \%$ (Africa), $19.2 \%$ (the Middle East), $10.5 \%$ to $12 \%$ [Oceania (Australia and New Zealand)], and $5.1 \%$ to $39.4 \%$ (Asia). The highest resistance reported for a single drug was for isoniazid [6]. This may be due to the liberal use of this drug for chemoprophylaxis.

For MDR-TB, the prevalence in Saudi Arabia for the period 1979-98 ranged from $1.5 \%$ to $44 \%$ and the highest resistance was for the drug combination of isoniazid and rifampicin. The WHO report on antituberculosis treatment [6] presented MDRTB rates that ranged from $0.2 \%$ to $1.1 \%$ (South America), 0\% to $18.1 \%$ (Europe), $0.9 \%$ to $7.3 \%$ (North America), $1.5 \%$ to $3.5 \%$ (Africa), $8.1 \%$ (the Middle East), $1 \%$ to 2\% [Oceania (Australia and New Zealand)] and $0.8 \%$ to $7.6 \%$ (Asia). Compared with these rates, the MDR-TB rate of $44 \%$, reported from the Gizan area of Saudi Arabia, was the highest rate overall. A possible explanation for this extremely high rate has been discussed earlier. Even when this high MDR-TB rate is excluded from the general picture, the MDR-TB rate in Saudi Arabia is still high, ranging from $1.5 \%$ to $25.7 \%$, thus placing Saudi Arabia firmly among those countries with the highest MDR-TB rates.

It is difficult to draw any strong conclusions about the status of drug-resistant TB in this country from these studies, for several reasons:

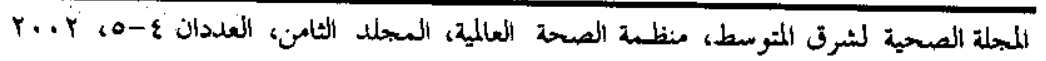


- Most of these reports were conducted mainly in regional hospitals (which might have different ratios of native to immigrant populations). Hospitals that treat high proportions of expatriates, who may be the source of highly-resistant tubercle bacilli, are likely to report high incidences of MDR-TB among their patients, as suggested by some of these studies.

- Some of the studies did not specify the site of $M$. tuberculosis isolation, that is pulmonary or extrapulmonary.

- Almost all of the studies failed to document patient compliance with treatment.

- Most studies failed to differentiate between primary and acquired resistance to TB in their study population.

- There was no standardized national TB treatment regimen.

- Some studies failed to document the method used for drug sensitivity testing. In addition, quality control between different laboratories is likely to have been variable, possibly affecting the accuracy of the results in some surveys.

Apart from possible variations in the populations studied, geographical origin of the patients, site of $M$. tuberculosis isolation (pulmonary or extrapulmonary), and patient compliance with treatment, the cause of this apparent discrepancy in prevalence of drug-resistant TB in different regions of Saudi Arabia remains unclear.

In conclusion, this review highlights the need to develop a standardized national policy for surveillance of drug-resistant TB in Saudi Arabia. This could be achieved by establishing a network of national reference laboratories, using internationally accepted and standardized methods of susceptibility testing. Such a system would allow trends in antituberculosis resistance over time to be monitored, in accordance with the WHO and the International Union Against Tuberculosis and Lung Disease (IUATLD) guidelines [3].

\section{References}

1. Dye $\mathbf{C}$ et al. Consensus statement. Giobal burden of tuberculosis: estimated incidence, prevalence, and mortality by country. WHO Global Surveiliance and Monitoring Project. Joumal of the American Medical Association, 1999, 282(7): 677-86.

2. Willcox PA. Drug-resistant tuberculosis: worldwide trends, problems specific to Eastern Europe and other hotspots, and the threat to developing countries. Current opinion in pulmonary medicine, 2001, 7(3):148-53.

3. Espinal MA et al. Global trends in resistance to antituberculosis drugs. World Health Organization, International Union against Tuberculosis and Lung Disease Working Group on Anti-tuberculo- sis Drug Resistance Surveillance. New England journal of medicine, 2001, 344(17):1294-303.

4. Cohn DL, Bustreo F, Raviglione MC. Drug-resistant tuberculosis: review of the worldwide situation and the WHO/ IUATLD Global Surveillance Project. Clinical infectious diseases, 1997, 24 (suppl.1):S121-30.

5. Pablos-Mendez $\mathrm{A}$ et al. Global surveillance for antituberculosis-drug resistance, 1994-1997. World Health Organization, International Union against Tuberculosis and Lung Disease Working Group on Anti-tuberculosis Drug Resistance Surveillance. New England journal of medicine, 1998, 338(23):1641-9. 
6. Anti-tuberculosis drug resistance in the world. Report 2: Prevalence and trends. Global project on anti-tuberculosis drug resistance surveillance. Geneva, World Health Organization, 2000:1-253 (document WHO/CDS/TB/2000.278).

7. Schwoebel $V$ et al. Standardization of anti-tuberculosis drug resistance surveillance in Europe. Recommendations of a World Health Organization (WHO) and International Union Against Tuberculosis and Lung Disease (IUATLD) Working Group. European respiratory journal, 2000, 16(2):364-71.

8. Al-Rubaish AM, Madania AA, AlMuhanna FA. Drug resistance pulmonary tuberculosis in the Eastern Province of Saudi Arabia. Saudi medical joumal, 2001, 22(9):776-9.

9. Wali $S$ et al. Multi-drug resistant tuberculosis. Saudi medical journal, 1998, 19: 802-6.

10. Al-Orainey 10 et al. Resistance to antituberculosis drugs in Riyadh, Saudi Arabia. Tubercle, 1989, 70(3):207-10.

11. Shanks NJ et al. Tuberculosis in Saudi Arabia. Saudi medical journal, 1983, 4:151-6.

12. Schiott $C R$ et al. Incidence of drug resistance amongst isolates of Mycobacterium tuberculosis recovered in Gizan area, Saudi Arabia. Saudi medical journal, 1985, 6:375-8.

13. Jarallah JS et al. High rate of rifampicin resistance of Mycobacterium tuberculosis in the Taif region of Saudi Arabia. Tubercle and lung disease, 1992, 73(2): 113-5.

14. Al-Orainey IO. Resistance to standard antituberculous drugs in Saudi Arabia. Saudi medical journal, 1989, 7:363-8.

15. Al-Rasheed AM et al. Low prevalence of multi-drug resistance tuberculosis in Saudi patients: an eleven-year retrospective study. A refereed abstract pre- sented at the 20th Annual Congress of the European Society of Mycobacteriology, Lucerne, Switzerland, 1999.

16. Ellis ME et al. High proportion of multidrug resistant Mycobacterium tuberculosis in Saudi Arabia. Scandinavian journal of infectious diseases, 1996, 28(6):591-5.

17. Zaman R. Tuberculosis in Saudi Arabia: initial and secondary drug resistance among indigenous and non-indigenous populations. Tubercle, 1991, 72(1):51-5.

18. Kinsara A et al. Review of tuberculosis at the King Khalid National Guard Hospital, Jeddah, Saudi Arabia. Saudi medical joumal, 1997, 19:212-4.

19. Khan MY et al. Increasing resistance of M. tuberculosis to anti-TB drugs in Saudi Arabia. International journal of antimicrobial agents, 2001, 17:415-8.

20. Milaat WA et al. Epidemiology of tuberculosis in Jeddah region, Saudi Arabia. Saudi medical joumal, 1994, 15:133-7.

21. Al-Zaeer A et al. Tuberculosis is the commonest cause of pneumonia requiring hospitalization during Hajj (pilgrimage to Makkah). Journal of infection, 1998, $36: 303-6$.

22. Al-Kassimi FA et al. Nationwide community survey of tuberculosis epidemiology in Saudi Arabia. Tubercle and lung disease, 1993, 74(4):254-60.

23. Al-Hajjaj MS, Al-Khatim IM. High rate of non-compliance with anti-tuberculosis treatment despite a retrieval system: a call for implementation of directly observed therapy in Saudi Arabia. International journal of tuberculosis and lung disease, 2000, 4(4):345-9.

24. Al-Kassimi FA. Review of tuberculosis in Saudi Arabia. Saudi medical journal, 1994, 15:192-5.

25. Rifai E. Tuberculosis in Arab countries. Saudi medical journal, 1982, 1:86-91. 